\title{
Lowland and Highland Varieties of Dioscorea esculenta Tubers Stimulate Growth of Lactobacillus sp. over Enterotoxigenic E. coli in vitro
}

\author{
Allan John R. Barcena, ${ }^{1}$ Aurora S. Nakpil, ${ }^{1}$ Nina G. Gloriani, MD, $\mathrm{PhD}^{2}$ and Paul Mark B. Medina, $\mathrm{PhD}^{3}$ \\ ${ }^{1}$ College of Medicine, University of the Philippines Manila \\ ${ }^{2}$ Department of Medical Microbiology, College of Public Health, University of the Philippines Manila \\ ${ }^{3}$ Biological Models Laboratory, Department of Biochemistry, Molecular Biology, College of Medicine, University of the Philippines Manila
}

\begin{abstract}
Objectives. Probiotic supplementation often only leads to transient improvement in the gut microbiome. Potential prebiotics, such as the oligosaccharide-rich varieties of Dioscorea esculenta tubers, can potentially bridge the gap between supplementation and persistent colonization. Thus, this study aimed to assess the ability of $D$. esculenta tubers to promote the growth of probiotic Lactobacillus sp. in vitro selectively.
\end{abstract}

Methods. The prebiotic activity of the selected varieties of Dioscorea esculenta tubers was evaluated via competitive growth assay, wherein the ratios of probiotic Lactobacillus sp. over enterotoxigenic Escherichia coli (ETEC) or "prebiotic ratios" were compared following treatment.

Results. Negative control $(0.9 \% \mathrm{NaCl}$ solution) produced a ratio of 0.88 , Lowland and Highland varieties produced ratios of 1.26 and 1.29, respectively, and positive control (inulin) produced 1.54. The two varieties had comparable ratios to one another $(p>0.05)$, and significantly higher ratios than the negative control $(p<0.05)$. Both varieties have significant prebiotic activity. Compared to inulin, the two varieties' prebiotic activity was $84 \%$ as effective.

Conclusion. Overall, the tubers promoted the growth of Lactobacillus sp. over ETEC. The crude tuber samples, given their availability and affordability, can be easily integrated into the local diet to contribute to the improvement of the general population's health.

Key Words: Dioscorea esculenta, Enterotoxigenic Escherichia coli, Inulin, Lactobacillus, Prebiotics

\section{INTRODUCTION}

Probiotics, broadly defined by the International Scientific Association for Probiotics and Prebiotics (ISAPP) as live microorganisms that when administered in adequate amounts confer a health benefit on the host, ${ }^{1}$ now constitute one of the most commonly consumed supplements globally. The increasing popularity of probiotic supplements largely emerges from a growing knowledge base about how probiotic

Poster presented at the $24^{\text {th }}$ International Union of Biochemistry and Molecular Biology Congress and $15^{\text {th }}$ Federation of Asian and Oceanian Biochemists and Molecular Biologists Congress on June 4-8, 2018, Seoul, South Korea

Corresponding author: Paul Mark B. Medina, PhD Biological Models Laboratory

Department of Biochemistry and Molecular Biology

College of Medicine

University of the Philippines Manila

547 Pedro Gil Street, Ermita, Manila 1000, Philippines

Email: pmbmedina@post.upm.edu.ph microorganisms can exert beneficial effects. Current studies suggest that probiotics, such as Lactobacillus sp. and Bifidobacterium sp., may confer benefits to the host through their interactions not only with the host's gut microbiota but also with the host's tissues. In vitro and in vivo studies have demonstrated that probiotics can promote resistance to pathogen colonization by competing for nutrients and epithelial attachment sites, ${ }^{2,3}$ by creating an acidic luminal environment, ${ }^{4}$ by producing antimicrobial ${ }^{5}$ and quorum quenching compounds, ${ }^{6-8}$ by improving the intestinal 
mucosa's barrier function, ${ }^{9}$ and by enhancing the host's innate and adaptive immune defenses. ${ }^{10,11} \mathrm{In}$ addition, it is claimed that probiotics possess the ability to produce systemic effects by producing bile acid hydrolases that promote cholesterol and triglyceride clearance, ${ }^{12,13}$ by attenuating key stress and anxiety-related pathways, ${ }^{14,15}$ and by modulating the host's overall immune response. ${ }^{10,11}$ Although various studies have shown that the intake of probiotics has improved outcomes in patients with infectious diarrhea, inflammatory bowel disease, inflammatory bowel syndrome, and necrotizing enterocolitis, there are trials with comparable methodological quality that suggest the opposite, collectively generating an inconsistent overall conclusion about the clinical efficacy of probiotic supplementation. ${ }^{16}$ Apart from limitations in methodology and data analysis, the conflicting results in literature may be attributed to individual differences in diet and baseline gut microbiome configuration, which are essential determinants of colonization. Several studies show that while supplementation leads to increased fecal probiotic concentration, it does not necessarily result in mucosal probiotic colonization. ${ }^{17,18}$ Additionally, the shedding of probiotic microorganisms in stool significantly diminishes following cessation of supplementation in the majority of hosts. ${ }^{19,20}$ One strategy that is hypothesized to bridge the gap between supplementation and persistent colonization is the consumption of prebiotics, defined by the ISAPP as substrates that are selectively utilized by host microorganisms conferring a health benefit. ${ }^{21}$ Typically composed of oligosaccharides, prebiotics may be fermented by probiotics, and in the process, provide a selectively nutritive environment for both probiotic microorganisms and the healthy gut microbiota. It is postulated that a diet with a suitable prebiotic composition can increase the likelihood of persistent probiotic gut colonization, potentially eliminating even the need for continuous probiotic supplementation. Edible tubers, such as yams of genus Dioscorea, form oligosaccharide-rich storage organs that may easily be integrated into an individual's regular diet to achieve an optimum dietary prebiotic intake. It has been shown that inulin, an oligosaccharide with an established prebiotic activity, may be extracted from Dioscorea esculenta tubers sampled from East Java. ${ }^{22}$ However, there have been no studies on the prebiotic activity of $D$. esculenta tubers when consumed as a whole. Furthermore, the prebiotic potential of the Philippine varieties of the D. esculenta remains scantily explored. D. esculenta, locally known as tugi, has two known locally cultivated varieties, Lowland and Highland, which produce tubers that differ in their morphological and chemical profiles. While both varieties of tubers are comparably rich in complex carbohydrates, the tubers of the Highland variety have been found to have higher total phenolic content. ${ }^{23}$ This becomes relevant considering the most recent consensus statement of ISAPP on the definition and scope of prebiotics where plant phenols, along with polyunsaturated fatty acids, have been named candidate prebiotics because several health benefits have been associated with the metabolites produced by their biotransformation by the gut microbiota. ${ }^{21}$ Yams are relatively easy to cultivate, and they remain to be a staple source of carbohydrates in rural areas across the country. The assessment of the prebiotic activity of D. esculenta tubers could provide a basis for the integration of edible tubers in the regular diet with the intent to provide a cost-effective strategy for the maintenance of healthy gut microbiome.

\section{METHODS}

\section{Processing and in vitro digestion}

The tubers were oven-dried and pulverized. Then for each variety, 5 grams of dry powder were dissolved in 90 $\mathrm{mL}$ of $0.9 \%$ saline solution. The generated solutions were subjected to in vitro digestion based on a protocol by Kho et al. ${ }^{12}$ Initially, $6 \mathrm{~mol} / \mathrm{L} \mathrm{HCl}$ was added until a $\mathrm{pH}$ of 2.0 was reached, then the solutions were left to stand for 15 minutes. Following the addition of $0.01 \mathrm{~g}$ pepsin, the solutions were left to stand for 2 hours. Through the addition of $1 \mathrm{~mol} / \mathrm{L}$ $\mathrm{NaHCO}_{3}$, the $\mathrm{pH}$ was adjusted to 5.0. The solutions were left to stand for another 2 hours after the addition of $0.0025 \mathrm{~g}$ pancreatin and $0.015 \mathrm{~g}$ bile salts. Finally, the $\mathrm{pH}$ was adjusted to 7.2 , using $0.5 \mathrm{~mol} / \mathrm{L} \mathrm{NaOH}$. Inulin powder and normal saline solution (NSS) were processed similarly to act as positive control and negative control, respectively.

\section{Bacterial strains}

Lactobacillus sp., the probiotic bacteria for this study, was isolated from a commercial product via direct plating on Lactobacillus MRS Agar. At the same time, the enterotoxigenic E. coli (ETEC), the growth competitor, was obtained from the culture collection of the Department of Medical Microbiology, College of Public Health, University of the Philippines Manila. The isolated strains underwent biochemical testing and selective plating onto MacConkey agar and Eosin Methylene Blue agar for identity confirmation.

\section{Determination of optimal dosage}

Enterotoxigenic $E$. coli were inoculated in LB broth, and the processed yams were added in varying concentrations $(1 \%-5 \% \mathrm{w} / \mathrm{v}$ in increments of $1 \%)$ to determine the amount of sample to be used for the competitive growth assay. The set-ups were incubated at $37^{\circ} \mathrm{C}$ for 48 hours, and enterotoxigenic $E$. coli colony forming units (CFUs) were quantified using the drop plate method on MacConkey agar plates. The ability of the yam samples to create a more favorable environment for the probiotic bacteria even when the conditions set were initially favorable for the pathogenic competitor provides a better measure of their prebiotic activity. Thus, concentrations that are expected to permit the optimal growth for the enterotoxigenic $E$. coli should be chosen. 


\section{Competitive growth assay}

Lactobacillus and enterotoxigenic E. coli were inoculated into LB broth preparations containing in vitro digested samples. Inulin powder acted as the positive control, while NSS alone acted as negative control. Initial CFU ratios of the prebiotic Lactobacillus versus the enterotoxigenic E. coli were adjusted to $1: 1$, then prebiotic activity was ascertained by comparing the CFU ratios after treatment. Set-ups were incubated at $37^{\circ} \mathrm{C}$ for 48 hours. Lactobacillus CFUs were quantified using the drop plate method on MRS agar plates, while MacConkey agar plates were used for enterotoxigenic E. coli.

\section{Data analysis}

Statistical analyses were performed on SPSS Statistics. A two-way analysis of variance (ANOVA) followed by Tukey's post-hoc test was used to determine statistically significant differences between and among treatments.

\section{RESULTS}

\section{Optimal dosage for the growth of ETEC is at least $2 \% \mathrm{w} / \mathrm{v}$}

The logarithmic values of $\mathrm{CFU} / \mathrm{mL}$ for $1 \% \mathrm{w} / \mathrm{v}$ sample concentration for both Lowland and Highland varieties were significantly lower $(p<0.05)$ than all other concentrations tested. There were no significant differences between and among $2 \%$ to $5 \% \mathrm{w} / \mathrm{v}$ sample concentrations, implying that the effect of the increasing concentration of sample added plateaued at $2 \% \mathrm{w} / \mathrm{v}$. Due to the induction of a set bacterial $\log$ phase, growth may plateau regardless of the amount of excess substrate due to the accumulation of toxic byproducts that can hinder the initially rapid growth of the bacterial population. To provide a favorable condition for the pathogenic competitor, the concentration was chosen for the competitive growth assay was $2 \% \mathrm{w} / \mathrm{v}$.

\section{ETEC CFU $/ \mathrm{mL}$ is highest in both $D$. esculenta tuber varieties}

The logarithmic value of $\mathrm{CFU} / \mathrm{mL}$ of enterotoxigenic $E$. coli was found to be highest in both varieties of $D$. esculenta tubers. There was no significant difference between the two varieties, but both significantly increased $(p<0.05)$ the logarithmic value of ETEC CFU/mL by $23 \%$ compared to the negative control (normal saline solution). Additionally, positive control (inulin) significantly reduced $(p<0.05)$ ETEC CFU/mL by $33 \%$ compared to the negative control. This reaffirms the prebiotic activity of inulin, which is the main ingredient in commercial prebiotics.

\section{Lactobacillus sp. CFU/mL is highest in both $D$. esculenta tuber varieties}

The colony count of Lactobacillus sp. was also found to be highest in both varieties of the D. esculenta tubers. There was no significant difference between the two tubers, and

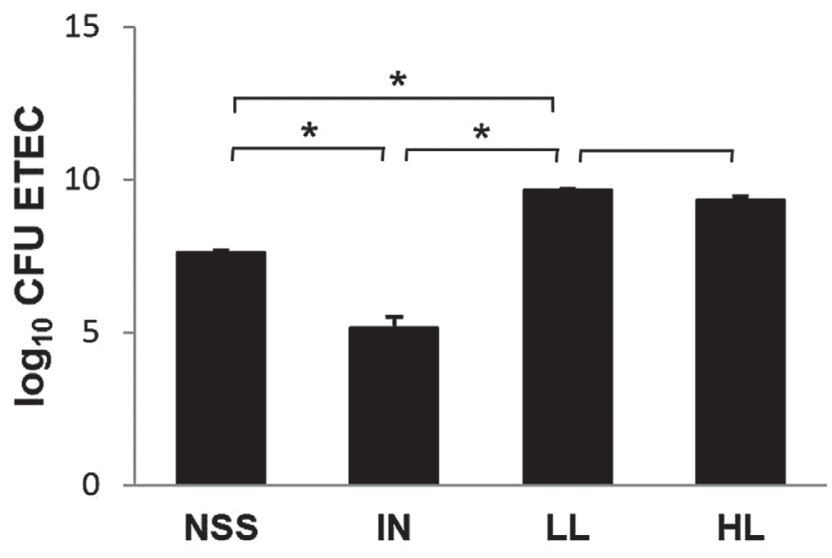

Figure 1. ETEC colony counts after 48 hours of incubation with different samples. This assay was performed in triplicate.

NSS - normal saline solution (0.9\%); IN - $1 \% \mathrm{w} / \mathrm{v}$ inulin; LL $2 \% \mathrm{w} / \mathrm{v}$ - Lowland variety; HL - $2 \% \mathrm{w} / \mathrm{v}$ - Highland variety.

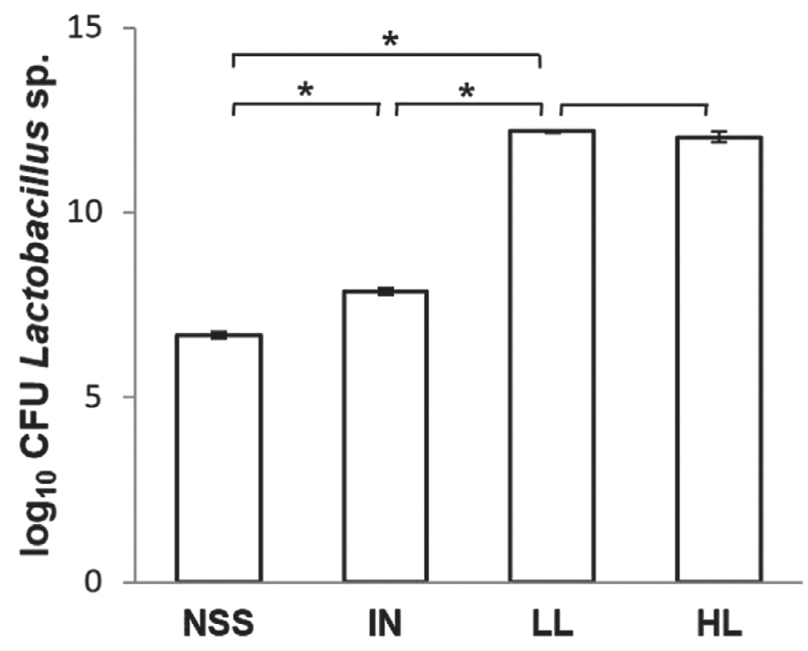

Figure 2. Lactobacillus sp.colony counts after 48 hours of incubation with different samples. This assay was performed in triplicate.

NSS - normal saline solution (0.9\%); IN - $1 \% \mathrm{w} / \mathrm{v}$ inulin; LL $2 \% \mathrm{w} / \mathrm{v}$ - Lowland variety; HL - $2 \% \mathrm{w} / \mathrm{v}$ - Highland variety.

both significantly increased $(p<0.05)$ the logarithmic value of Lactobacillus sp. CFU/mL by more than $80 \%$ compared to the negative control. The positive control only increased the logarithmic value of Lactobacillus sp. CFU/mL by $18 \%$ versus the negative control $(p<0.05)$.

\section{Both Lowland and Highland tuber varieties showed prebiotic activity that is at least $84 \%$ as effective as inulin}

Across all treatment conditions, the growth of Lactobacillus sp. and ETEC were significantly different from one another $(p<0.05)$. For the negative control, there was a higher number of ETEC colonies, and this was expected 


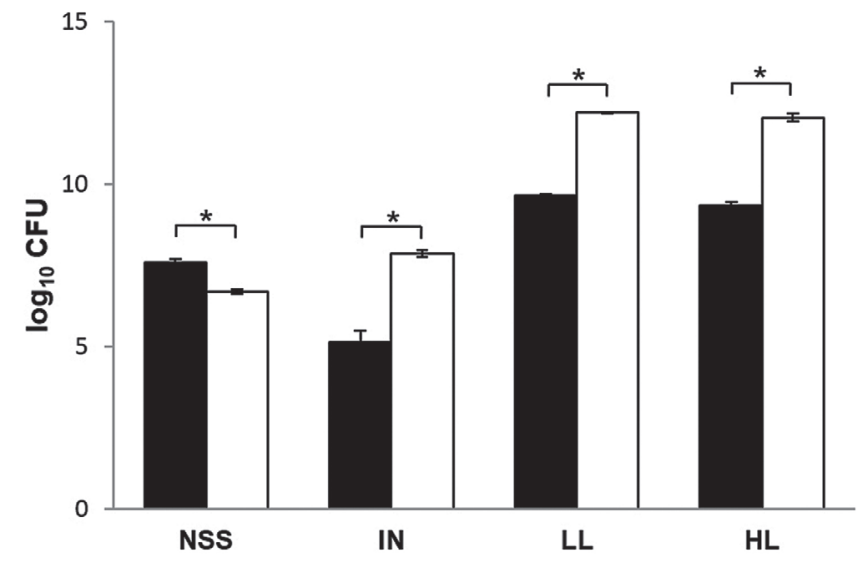

Figure 3. Comparison of Lactobacillus species (White) and ETEC (Black) colony counts after 48 hours of incubation with different samples. This assay was performed in triplicate.

NSS - normal saline solution (0.9\%); IN - 1\% w/v inulin; LL $2 \% \mathrm{w} / \mathrm{v}$ - Lowland variety; $\mathrm{HL}-2 \% \mathrm{w} / \mathrm{v}$ - Highland variety.

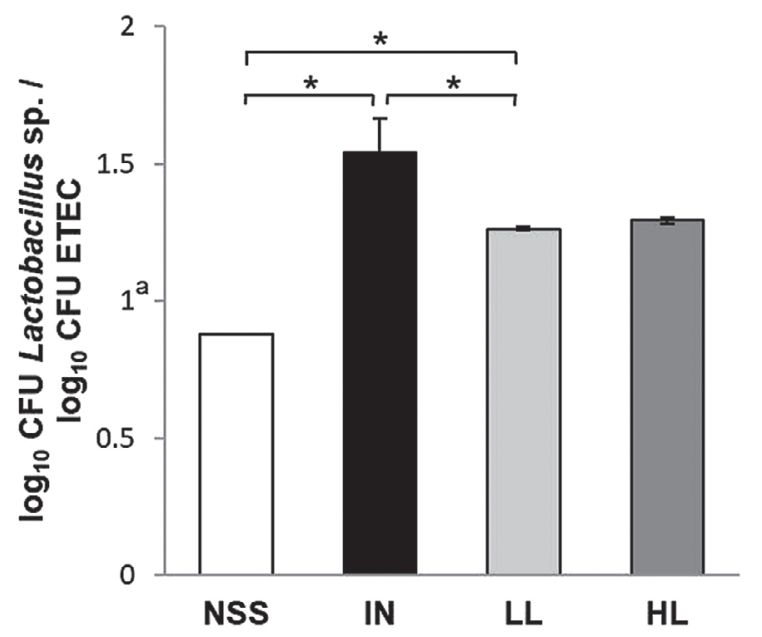

Figure 4. Growth ratios of Lactobacillus species over ETEC after 48 hours of incubation with different samples. This assay was performed in triplicate.

NSS - normal saline solution (0.9\%); IN - 1\% w/v inulin; LL $2 \% \mathrm{w} / \mathrm{v}$ - Lowland variety; HL - $2 \% \mathrm{w} / \mathrm{v}$ - Highland variety. ${ }^{a}$ Starting ratio of Lactobacillus sp. and ETEC.

as the primary conditions were optimized for the growth of ETEC. For the positive control, and both the Lowland and Highland variety treatments, there was a significantly larger amount of Lactobacillus sp. colonies $(p<0.05)$.

To evaluate the efficacy of each treatment condition in promoting the growth of the probiotic Lactobacillus sp. over enterotoxigenic $E$. coli, the ratios between the logarithmic values of bacterial $\mathrm{CFU} / \mathrm{mL}$ per treatment condition were compared. Ratios were calculated as $\log$ Lactobacillus sp. $\mathrm{CFU} / \mathrm{mL} / \log$ ETEC CFU/mL. A prebiotic ratio of 1 reflects an equal number of colonies for both types of bacteria, and a value greater than 1 is indicative of greater amounts of Lactobacillus sp. than ETEC, and thus, prebiotic activity. Negative control $(0.9 \% \mathrm{NaCl}$ solution) produced a ratio of 0.88, Lowland and Highland varieties produced ratios of 1.26 and 1.29, respectively, and positive control (inulin) produced a ratio of 1.54 . The two varieties had comparable prebiotic ratios to one another $(p>0.05)$, and significantly greater prebiotic ratios than the negative control $(p<0.05)$. This indicates that both varieties of $D$. esculenta tubers have overall prebiotic activity. Although both variety treatments had larger absolute counts of Lactobacillus sp. colonies than the positive control, the ratios show that positive control still possesses the best prebiotic activity as both variety treatments had a significantly lower $(p<0.05)$ ratio than the positive control. When compared with the positive control, inulin, the two varieties' prebiotic activity was at least $84 \%$ as effective.

\section{DISCUSSION}

The effect of the D. esculenta tubers on the absolute colony counts of both ETEC and Lactobacillus sp. suggests that both varieties contain substances that are non-selectively nutritive. One such substance is starch, which may constitute up to $80 \%$ of the tubers' dry weight. ${ }^{24}$ Starch extracted from D. esculenta tubers was shown to have a relatively smaller granule size, which is associated with the greater release of glucose with more of the surface area of the substrate exposed for the number of residual macronutrients enzymatic breakdown during digestion. ${ }^{25}$ Nevertheless, the tubers were able to produce a significant overall prebiotic effect in vitro even when the treatment solutions have likely retained significant amounts of digested starch. This supports the idea that the selectively nutritive oligosaccharides, which are only available to Lactobacillus sp. for utilization, play a bigger role in the probiotic-competitor interaction under study. More importantly, it may be inferred that the inhibitory effect that probiotics and their metabolic byproducts can impose may negate the beneficial effects that can be provided by nonselectively nutritive substrates to pathogenic competitors, such as ETEC. As mentioned previously, the presence of an adequate supply of prebiotic substrates allows probiotics to proliferate and to subsequently inhibit the growth of pathogenic microorganisms through a diverse array of mechanisms. The ratio of selectively nutritive and nonselectively nutritive substances that is most optimal for the persistent colonization of probiotic microorganisms remains unknown, and further investigations are necessary to elucidate how the presence and the quantity of remaining macronutrients in the gut will affect the action of prebiotics upon supplementation. With this, the prebiotic activity of the tubers is hypothesized to improve in vivo. Given that the samples have only undergone in vitro digestion and not intestinal absorption, it is expected that, in vivo, a significant fraction if not most of the digested starch that the tubers contain will be absorbed by the host, leaving only the 
indigestible substances such as fermentable oligosaccharides for the gut microorganisms. The expected reduction in non-selectively nutritive substances will make it less likely for the ETEC population to catch up with the growth of Lactobacillus sp., thus, potentially increasing the overall prebiotic effect of the tubers. While the performance of the tubers may even improve in vivo, more research is needed to confirm this hypothesis and to factor in the multiplicity of determinants that influence the overall composition of the gut microbiota. The result of the competitive growth assay is limited to the interaction between ETEC and Lactobacillus sp. in a controlled environment, and the reproducibility of this result in an environment with other gut microorganisms has yet to be determined. The current opinion concerning balance in the host's gut microbiome is that it largely depends on the ratio of microorganisms colonizing the intestinal tract. ${ }^{26}$ However, the role of absolute bacterial counts remains poorly understood. Currently, there are no clear-cut genera or species-specific limits as to what constitutes undergrowth or even overgrowth in the gastrointestinal tract, and the differential effect of substances that may produce the similar prebiotic ratios on the absolute count of both probiotics and pathogenic competitors needs further exploration. More importantly, further studies are needed to ultimately identify the impact of the tubers on both mucosal probiotic colonization and persistence post-supplementation.

Even if it has been shown previously that inulin constitutes only up to $20 \%$ of D. esculenta tubers' dry weight, both tuber varieties used in this study at $2 \% \mathrm{w} / \mathrm{v}$ have remarkably demonstrated a prebiotic activity that is at least $84 \%$ as effective as $1 \% \mathrm{w} / \mathrm{v}$ pure inulin. This implies that despite having an estimated inulin composition that is less than half of that of the positive control, the tubers have produced a comparable activity. This provides a potential lead to the role of other known and unknown substances found in the tubers in selectively promoting the growth of probiotic microorganisms. Previous studies have reported that Dioscorea tubers contain significant amounts of substances now considered as candidate prebiotics, such as phenolic compound ${ }^{23,27}$ and polyunsaturated fatty acids. ${ }^{28}$ However, the extent of their contribution to the tubers' overall prebiotic activity remains unknown. Polyunsaturated fatty acids are thought to exert their prebiotic effect by providing a substrate to produce short-chain fatty acids, such as acetate and butyrate, which are essential mediators of several mechanisms by which probiotics provide beneficial effects, including the improvement of the host's intestinal epithelial barrier integrity and the activation of the host's immune response to invading infectious agents and even malignant cells. ${ }^{29,30}$

On the other hand, the majority of dietary polyphenols remain undigested after passing the small intestine and, therefore, provide substrates for probiotic microorganisms in the colon that may be converted into metabolites that may benefit the host. ${ }^{31}$ While it has been shown that the tubers of the Highland variety have higher total phenolic content, ${ }^{23}$ the two varieties had comparable prebiotic activity. Thus, it may be inferred that the phenols found in the tubers play a small role in promoting the growth of Lactobacillus sp. over ETEC. Aside from the well-known inulin, fructooligosaccharides, galactooligosaccharides, mannanoligosaccharides, and xylooligosaccharides may also be present in the tubers of D. esculenta, However, their contribution to the tubers' oligosaccharide composition needs to be confirmed. However, like phenolic compounds and polyunsaturated fatty acids, most of the previously mentioned oligosaccharides require more studies in human subjects to confirm their effects and to eventually fulfill their prebiotic status. ${ }^{16}$

This study has demonstrated the prebiotic activity of the tubers of both Highland and Lowland varieties of D. escuenta in vitro. While this necessitates confirmation in more complex in vivo models, the results allude to the possible utility of the tubers themselves as effective prebiotic supplements in place of isolating the specific known and candidate prebiotic compounds, such as oligosaccharides, phenols, and polyunsaturated fatty acids, before supplementation. This hypothesis provides a foundation for a more cost-effective and more convenient approach to the integration of prebiotic substances into an individual's diet. More importantly, it provides a basis for the investigation of simple dietary modification to promote and sustain persistent mucosal probiotic colonization.

\section{CONCLUSION}

Overall, the tubers promoted the growth of Lactobacillus sp. over ETEC, and this is confirmed by a comparison of the prebiotic ratios of the different treatments. While the positive control still produced the highest prebiotic ratio (1.54), the Lowland (1.26) and Highland (1.29) varieties were at least $84 \%$ as effective despite being administered as crude treatments. This allows for the utilization of the tubers' potential health benefits without the need for sophisticated purification methods. Easy access by the general population to the product due to their wide availability and relatively low price makes $D$. esculenta tubers a simple integration into the general diet. The prebiotic activity of D. esculenta tubers is mostly attributed to their relatively high inulin content. However, given the crudeness of the samples, there may be other substances that can explain their remarkable effects on the growth of probiotic species such as Lactobacillus. Thus, further characterization of the D. esculenta tubers should pave the way for a better understanding of not only how these tubers exert their prebiotic effect but also other potential health benefits of consuming these tubers.

\section{Acknowledgment}

This project was supported by the Department of Biochemistry and Molecular Biology, College of Medicine, University of the Philippines Manila, and the Depart- 
ment of Medical Microbiology, College of Public Health, University of the Philippines Manila.

\section{Statement of Authorship}

Mr. Allan John R. Barcena and Ms. Aurora S. Nakpil - these two authors contributed equally to this research work. All authors have approved the final version submitted.

\section{Author Disclosure}

All authors have declared no conflict of interest.

\section{Funding Source}

This research did not receive any specific grant from funding agencies in the public, commercial, or not-forprofit sectors.

\section{REFERENCES}

1. Hill C, Guarner F, Reid G, Gibson GR, Merenstein DJ, Pot B, et al. Expert consensus document: The International Scientific Association for Probiotics and Prebiotics consensus statement on the scope and appropriate use of the term probiotic. Nature Reviews: Gastroenterology \& Hepatology. 2014; 11:506-14.

2. Gueimonde M, Margolles A, de los Reyes-Gavilán CG, Salminen S. Competitive exclusion of enteropathogens from human intestinal mucus by Bifidobacterium strains with acquired resistance to bile- a preliminary study. International Journal of Food Microbiology. 2007; 113:228-32.

3. Kim Y, Kim SH, Whang KY, Kim YJ, Oh S. Inhibition of Escherichia coli O157:H7 attachment by interactions between lactic acid bacteria and intestinal epithelial cells. Journal of Microbiology and Biotechnology. 2008; 18:278-1285.

4. Ríos-Covián D, Ruas-Madiedo P, Margolles A, Gueimonde M, de Los Reyes-Gavilán CG, Salazar N. Intestinal short chain fatty acids and their link with diet and human health. Frontiers in Microbiology. 2016; 7:185.

5. Bali V, Panesar PS, Bera MB, Kennedy JF. Bacteriocins: recent trends and potential applications. Critical Reviews in Food Science and Nutrition. 2016; 56:817-34.

6. Medellin-Peña MJ, Wang H, Johnson R, Anand S, Griffiths MW. Probiotics affect virulence-related gene expression in Escherichia coli O157:H7. Applied and Environmental Microbiology. 2007; 73: 4259-67.

7. Yun B, Oh S, Griffiths MW. Lactobacillus acidophilus modulates the virulence of Clostridium difficile. Journal of Dairy Science. 2014; 97: 4745-58.

8. Li J, Wang W, Xu SX, Magarvey NA, McCormick JK. Lactobacillus reuteri-produced cyclic dipeptides quench agr-mediated expression of toxic shock syndrome toxin-1 in staphylococci. Proceedings of the National Academy of Sciences of the United States of America. 2011; 108:3360-5.

9. Ohland CL, Macnaughton WK. Probiotic bacteria and intestinal epithelial barrier function. American Journal of Physiology: Gastrointestinal and Liver Physiology. 2010; 298:G807-G819.

10. Thomas CM, Versalovic J. Probiotics-host communication: modulation of signaling pathways in the intestine. Gut Microbes. 2010; 1:148-163.

11. Klaenhammer TR, Kleerebezem M, Kopp MV, Rescigno M. The impact of probiotics and prebiotics on the immune system. Nature Reviews: Immunology. 2012; 12:728-34.

12. Joyce SA, MacSharry J, Casey PG, Kinsella M, Murphy EF, Shanahan $\mathrm{F}$, et al. Regulation of host weight gain and lipid metabolism by bacterial bile acid modification in the gut. Proceedings of the National Academy of Sciences of the United States of America. 2014; 111: 7421-6.
13. Costabile A, Buttarazzi I, Kolida S, Quercia S, Baldini J, Swann $\mathrm{JR}$, et al. An in vivo assessment of the cholesterol- lowering efficacy of Lactobacillus plantarum ECGC 13110402 in normal to mildly hypercholesterolaemic adults. PLoS One. 2017; 66.

14. Sarkar A, Lehto SM, Harty S, Dinan TG, Cryan JF, Burnet PWJ, et al. Psychobiotics and the manipulation of bacteria-gut-brain signals. Trends in Neurosciences. 2016; 39:763-81.

15. Kim N, Yun M, Oh YJ, Choi HJ. Mind- altering with the gut: modulation of the gut- brain axis with probiotics. Journal of Microbiology. 2018; 56:172-82.

16. Suez J, Zmora N, Segal E, Elinav E. The pros, cons, and many unknowns of probiotics. Nature Medicine. 2019; 25:716-29.

17. Goossens DA, Jonkers DM, Russel MG, Stobberingh EE, Stockbrügger RW. The effect of a probiotic drink with Lactobacillus plantarum $299 \mathrm{v}$ on the bacterial composition in faeces and mucosal biopsies of rectum and ascending colon. Alimentary Pharmacology \& Therapeutics. 2006; 23:255-63.

18. Zmora N, Zilberman-Schapira G, Suez J, Mor U, Dori-Bachash $\mathrm{M}$, Bashiardes S. Personalized gut mucosal colonization resistance to empiric probiotics is associated with unique host and microbiome features. Cell. 2018; 174:1388-1405.

19. Frese SA, Hutkins RW, Walter J. Comparison of the colonization ability of autochthonous and allochthonous strains of lactobacilli in the human gastrointestinal tract. Advanced Microbiology. 2012; 399.

20. Charbonneau D, Gibb RD, Quigley EM. Fecal excretion of Bifidobacterium infantis 35624 and changes in fecal microbiota after eight weeks of oral supplementation with encapsulated probiotic. Gut Microbes. 2013; 4:201-11.

21. Gibson GR, Hutkins R, Sanders ME, Prescott SL, Reimer RA, Salminen SJ, et al. Expert consensus document: The International Scientific Association for Probiotics and Prebiotics (ISAPP) consensus statement on the definition and scope of prebiotics. Nature Reviews Gastroenterology \& Hepatology. 2017; 14(8):491-502.

22. Winarti S, Harmayani E, Marsono Y, Pranoto Y. Effect of inulin isolated from lesser yam (Dioscorea esculenta) on the growth of probiotics bacteria and SCFA formation during fermentation. International Journal of Microbiology. 2013; 4(2):55-63.

23. Kho MJN, Hernandez RBB, Ladera JPT, Khow DAT, Lagura VAM, Jacinto JAC, Medina PMB. In vitro assessment of the prebiotic potential of Caulerpa lentillifera, Gracilaria arcuata, and Sargassum polycystum. International Journal of Bioscience. 2017; 10(5):382-88.

24. Senanayake S, Ranaweera K., Bamunuarachchi A, Gunaratne A. Proximate analysis and phytochemical and mineral constituents in four cultivars of yams and tuber crops in Sri Lanka. Tropical Agricultural Research and Extension. 2013; 15(1):32.

25. Teo ML, Small DM. The effect of granule size on the digestibility of wheat starch using an in vitro model. International Journal of Nutrition and Food Engineering. 2012; 6(9):756-60.

26. Rinninella E, Raoul P, Cintoni M, Franceschi F, Miggiano G, Gasbarrini A, Mele M. What is the Healthy Gut Microbiota Composition? A Changing Ecosystem across Age, Environment, Diet, and Diseases. Microorganisms. 2019; 7(1):14.

27. Cornago DF, Rumbaoa RO, Geronimo IM. Philippine yam (Dioscorea spp.) tubers phenolic content and antioxidant capacity. Philippine Journal of Science. 2011; 140(2):145-52.

28. Kouassi B, Diopoh J, Leroy Y, Fournet B. Total amino acids and fatty acids composition of yam (Dioscorea) tubers and their evolution during storage. Journal of the Science of Food and Agriculture. 1988; 42(3):273-85.

29. Canfora EE, Jocken JW, Blaak EE. Short-chain fatty acids in control of body weight and insulin sensitivity. Nature Reviews: Endocrinology. 2015; 11:577-91.

30. Koh A, De Vadder F, Kovatcheva-Datchary P, Backhed F. From dietary fiber to host physiology: short-chain fatty acids as key bacterial metabolites. Cell. 2016; 165:1332-45.

31. Clifford MN. Diet-derived phenols in plasma and tissues and their implications for health. Planta Medica. 2004; 70:1103-14. 